\title{
Effects of reionization on dwarf galaxy formation
}

\author{
Massimo Ricotti ${ }^{1}$ \\ ${ }^{1}$ Department of Astronomy, University of Maryland at College Park, College Park, MD 20742 \\ email: ricotti@astro.umd.edu
}

\begin{abstract}
In this talk I revisit the problem of gas accretion onto minihalos after reionization. I show that primordial minihalos with $v_{c i r}<20 \mathrm{~km} \mathrm{~s}^{-1}$ stop accreting gas after reionization, as is usually assumed, but in virtue of their increasing concentration and the decreasing temperature of the intergalactic medium as redshift decreases, they have a late phase (at redshift $z<2$ ) of gas accretion and possibly star formation. As a result we expect that pre-reionization fossils have a more complex star formation history than previously envisioned. A signature of this model is a bimodal star formation history. The dwarf spheroidal galaxy Leo $\mathrm{T}$, that inspired the present work, fits with this scenario. Another prediction of the model is the existence of a population of gas rich minihalos that never formed stars. A subset of compact high-velocity clouds may be identified as such objects but the bulk of them may still be undiscovered.
\end{abstract}

Keywords. galaxies: dwarf, galaxies: evolution, Local Group, early universe

\section{Introduction}

It is widely believed that reionization of the intergalactic medium (IGM) suppresses gas accretion and galaxy formation in small halos with circular velocity $v_{\text {cir }}<20 \mathrm{~km} \mathrm{~s}^{-1}$, corresponding to a virial temperature $T_{v i r} \lesssim 20,000 \mathrm{~K}$ and to dark halo masses $M \lesssim 10^{8}-$ $10^{9} \mathrm{M}_{\odot}$ (e.g., Babul \& Rees 1992; Efstathiou 1992). After reionization, the Jeans mass in the IGM exceeds the mass of these minihalos and the IGM gas is unable to condense under the influence of their gravitational potential. However, a recently discovered dwarf spheroidal (dSph) galaxy - Leo T (Ryan-Weber et al. 2008) - despite having an estimated mass of about $7 \times 10^{6} \mathrm{M}_{\odot}$, contains gas and is actively forming stars at the present day. Leo $\mathrm{T}$ is one of the smallest dSphs in the Local Group and is located in the outskirts of the Milky Way at a distance of about $400 \mathrm{kpc}$, that makes it unlikely to be tidally stripped. It is possible that the total mass of Leo $\mathrm{T}$ is much larger than observed because Leo $\mathrm{T}$ dark halo extends well beyond the stellar and gas radii. But Leo $\mathrm{T}$ properties are very similar to those of ultra-faint $\mathrm{dSph}$ galaxies and differ from those of more massive dIrr galaxies that are able to hold on to their gas. The puzzling observation of Leo T inspired us at taking a fresh look at the problem of gas accretion onto minihalos after reionization. The results of our study are independent of the existence of Leo $\mathrm{T}$ and are fairly general.

In this talk we note that the ability of a halo to accrete gas does not depend solely on the ratio of their mass to the IGM Jeans mass but also on the concentration, $c$, of the dark halo. Typically, the concentration of a halo is $c_{v i r} \sim 5$ at the redshift of formation but, as the halo evolves (in relative isolation) in the expanding universe its concentration increases. This is expected as a result of cosmological secondary infall of dark matter and gas from the IGM onto the virialized halo (Bertschinger 1985) and is confirmed by Nbody simulations (Bullock et al. 2001; Wechsler et al. 2002). In this contribution we show that primordial minihalos with $v_{c i r}<20 \mathrm{~km} \mathrm{~s}^{-1}$ stop accreting gas after reionization, as 
expected, but in virtue of their increasing concentration and the decreasing temperature of the IGM at $z<3$, they start accreting gas again at later times. Thus, we expect that a few pre-reionization fossils (Ricotti \& Gnedin 2005, hereafter RG05) in the Local Group, most likely those that evolved on the outskirts of the Milky Way until recently, have a bimodal star formation history with an old $(\sim 13 \mathrm{Gyr})$ and a younger $(\lesssim 5-10 \mathrm{Gyr}$, depending on the minihalo mass) population of stars.

Another prediction of the model is the possible existence of minihalos containing only gas but no stars. This would revive the suggestion that some compact high-velocity clouds (HVCs) are extragalactic objects (Blitz et al. 1999).

\section{Evolution of Gas density profile}

N-body simulations show that the concentration of dark halos is, on average, $c(z) \propto$ $(1+z)^{-1}$ (Bullock et al. 2001; Wechsler et al. 2002). At virialization the concentration of halos is $c_{v i r} \sim 5$. Assuming that halos have a universal NFW density profile, $c_{v i r}$ has a weak dependence on the halo mass, or, if the halo profile has an inner slope that depends on the halos mass (Ricotti 2003; Ricotti \& Wilkinson 2004; Ricotti et al. 2007), $c_{v i r}$ is indeed a universal constant. Either way, although with some scatter and a marked dependence on the environment, the mean halo concentration is $c(z)=c_{v i r}\left(1+z_{v i r}\right) /(1+$ $z$ ), where $z_{v i r}$ depends on $v_{\text {cir }}$ and the cosmology. Small mass halos form earlier than larger ones and typically have a larger concentration at $z=0$. It is important to consider the dependence of the halo concentration on the environment. An important requirement of our model is that the minihalo forms at high-z and evolves in a low or a mean density region of the universe, in order to maintain a large concentration at $z=0$.

For a given value of the parameter $\Gamma=\left(v_{c i r} / c_{s}\right)^{2}=T_{v i r} / T$, the density profile of the gas in a minihalo depends on the concentration of the dark halo and on the ability of the gas to recombine and cool. Figure 1 (left panel) shows the gas overdensity profile for an isothermal (solid curves) and an adiabatic (dotted curves) gas in hydrostatic equilibrium in NFW halos with different values of the concentration. If the gas is adiabatic the overdensity in the core does not exceed 100. However, if the gas condenses isothermally, the overdensity can reach values of $10^{9}$.

The recombination time in the IGM is longer then the Hubble time at any redshift $z \lesssim 14$, thus, once hydrogen in the IGM becomes fully ionized, it remains ionized even if the ionization background drops to zero and the temperature evolution is nearly adiabatic. Similarly, when He II reionizes at $z \sim 3$, the IGM becomes hotter, but after that the IGM temperature evolves almost adiabatically. The gas inside a minihalo initially evolves with the same equation of state (EOS) as the IGM. When, due to adiabatic compression, the overdensity becomes larger than a critical value at which the recombination time scale, $t_{r e c}$ becomes shorter than the Hubble time $t_{H}$, the gas can recombine and cool.

The cooling time for a gas with $T>10^{4} \mathrm{~K}$ is always $t_{c o o l}<t_{r e c}$, independently of the assumed ionization fraction, temperature and density of the gas. Thus, if the gas can recombine it is also able to cool and its EOS becomes isothermal. The cooling is dominated by $\mathrm{H}$ and He recombination lines if the gas is highly ionized by UV radiation $\left(x_{e} \lesssim 10^{-3}\right)$, or $\mathrm{H}$ and He Lyman- $\alpha$ otherwise. Figure 1 (right panel) shows the evolution of the gas density (top panel) and $t_{r e c} / t_{H}$ (bottom panel) in the core of minihalos of different $v_{c i r}$ for isothermal and adiabatic gas. The EOS of the IGM at low redshift determines the critical circular velocity $v_{c i r}^{c r}(z)$ at which $t_{r e c} / t_{H}<1$. At $z=0$ the condition $t_{\text {rec }}=\left(n_{g, \text { core }} \alpha(T)\right)^{-1}<t_{H}$, can be written as $n_{g, \text { core }}>n_{g, \text { core }}^{c r} \sim 10^{-4} \mathrm{~cm}^{-3}$, or as $\beta(z)>1.5\left[(14 /(1+z)-1]\right.$. Thus, at $z=0, v_{\text {cir }}^{c r} \approx c_{s}^{I G M} 19.5 /(4.4+c / 4)$. Minihalos 

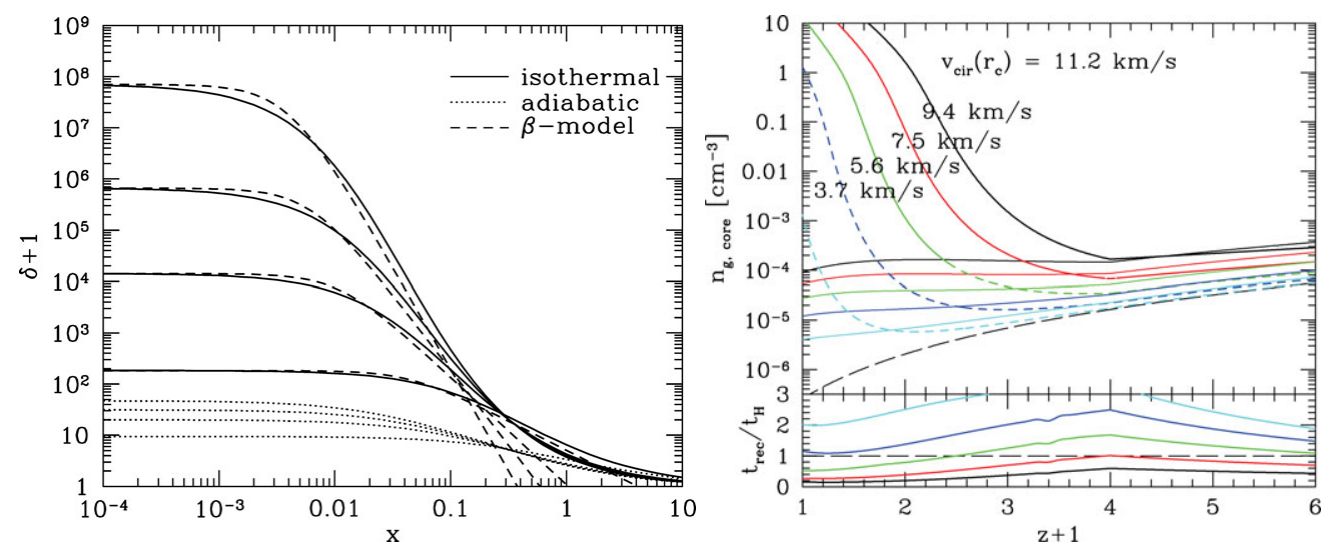

Figure 1. (Left). The evolution of the gas density in the core of dark halos for halos with $v_{c i r}=18,15,12,9$ and $6 \mathrm{~km} \mathrm{~s}^{-1}$ (from the top to the bottom). The thick curves assume an isothermal gas in the halo and the thin curves adiabatic. The curves are dashed if the gas cannot cool $\left(t_{r e c} / t_{H}>1\right)$ and solid otherwise $\left(t_{r e c} / t_{H}<1\right)$. The curves from the bottom to the top in the lower insert panel show $t_{r e c} / t_{H}$ as a function of redshift for the same halos as in the top panel. The redshift of virialization is $z_{v i r}=15$ for all halos.(Right). The overdensity profile of gas in hydrostatic equilibrium in a NFW dark matter halo virialized at $z_{v i r}=0,3,6$ and 10, (corresponding to halo concentrations, $c=5,20,35$ and 50), from bottom to top respectively. The solid and dotted curves are for isothermal and adiabatic equations of state, respectively. The $\beta$ - model fit of the isothermal profile is shown as a dashed line. All halos have $\Gamma=T_{v i r} / T=1$. The halo radii are normalized to unity.

with $v_{c i r}>v_{c i r}^{c r}$ condense isothermally and may have sufficiently large values of the core density and hydrogen column density to be observable by $21 \mathrm{~cm}$ and $\mathrm{H}-\alpha$ emission or in absorption. The isothermal density profile is well approximated by a $\beta$-model (see Fig. 1 , above) with core radius $r_{\text {core }} \approx 0.22 r_{s} \approx r_{v i r} / 20$. Approximately we have:

$$
r_{\text {core }} \approx 75 \mathrm{pc}\left(\frac{v_{\text {cir }}}{17 \mathrm{~km} / \mathrm{s}}\right)\left(\frac{10}{1+z_{\text {vir }}}\right)^{1.5},
$$

although, depending on the values of the core overdensity, lower density gas may extend to $r_{h} \approx 15 \mathrm{kpc}\left(M / 10^{8} M_{\odot}\right)^{1 / 3} /(1+z)$. The circular velocity at $r_{\text {core }}$ is

$$
v_{\text {cir }}\left(r_{\text {core }}\right) \approx 0.66 v_{\text {cir }} \approx 0.624 v_{\text {vir }}^{\max } \text {. }
$$

The accreted gas mass does not exceed $M_{g} \approx\left(\Delta_{g} / 178\right)\left(\Omega_{b} / \Omega_{d m}\right) M$, where $\Delta_{g} \sim 5$ is the mean gas overdensity in the halo. For core overdensities $\gtrsim 10^{6}$ most of the accreted gas is within the core, so the ratio of the dark matter mass and the gas mass at $r_{\text {core }}$ is $M / M_{g} \approx 235$, independent of redshift and halo parameters.

Minihalos that can accrete gas may not be able to form stars because the gas temperature is $\sim 10^{4} \mathrm{~K}$ if the gas is metal free. These halos may be stellarless as compact HVCs or may be pre-reionization fossils with an old stellar population and gas. In order to form stars the minihalo must cool to a lower temperature and develop a multi-phase ISM. The cooling by $\mathrm{H}_{2}$ in a metal free gas is not viable at $z=0$ due to the dominant $\mathrm{H}_{2}$ photo-dissociating background and absence of positive feedback processes (Ricotti et al. 2002). The cooling function from hyperfine transitions of oxygen and carbon depends on the gas metallicity, $Z$, roughly as $\Lambda_{23} \sim 10^{-3}\left(Z / Z_{\odot}\right)$, where $\Lambda_{23}=10^{-23} \mathrm{erg} \mathrm{s}^{-1} \mathrm{~cm}^{3}$. Thus, a necessary condition for star formation, $t_{\text {cool }} \approx 0.7 \mathrm{yr} T /\left(n_{g, \text { core }} \Lambda_{-23}\right)<t_{H}$, is

$$
n_{\text {g,core }}>n_{g, \text { core }}^{*} \sim 0.05 \mathrm{~cm}^{-3}\left(\frac{Z}{10^{-2} Z_{\odot}}\right)^{-1} \text {. }
$$



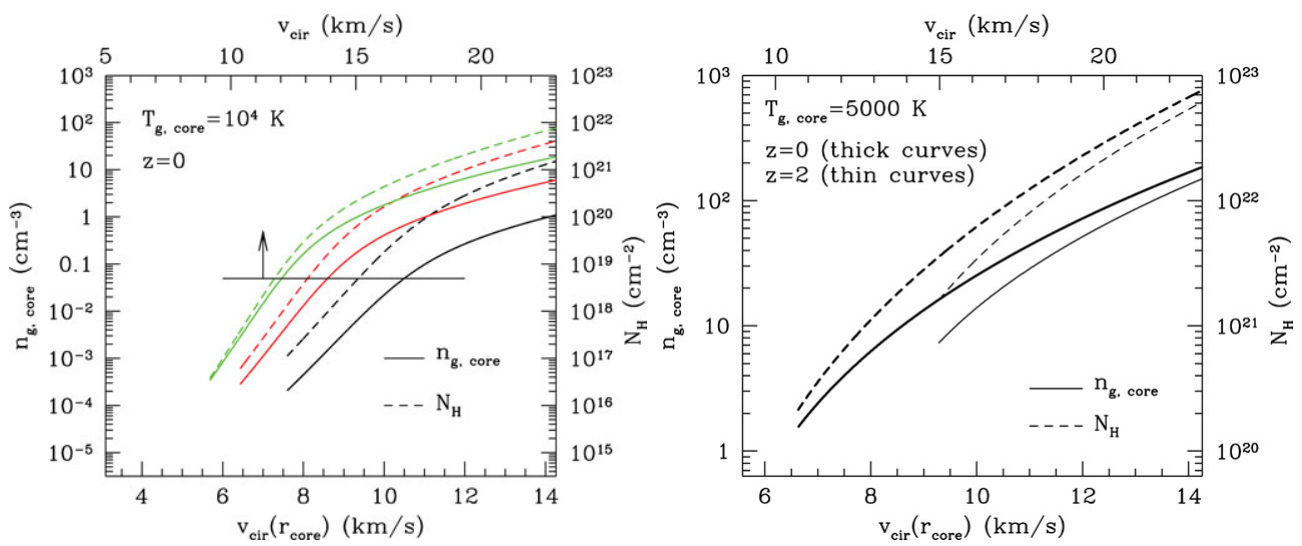

Figure 2. (left) The gas density, $n_{g, c o r e}$ (solid curves), and hydrogen column density, $N_{H}=2 r_{c} n_{g, c o r e}$ (dashed curves), within the core, $r_{c}$, of a minihalo as a function its circular velocity at $r_{c}$ at $z=0$. The curves from the bottom to the top refer to 2,3 and $4-\sigma$ perturbations. The minimum $v_{c i r}$ in each curve is determined by the condition $t_{r e c} / t_{H}<1$, necessary for cooling to $T_{\text {gas }} \sim 10^{4} \mathrm{~K}$. The horizontal line (with arrow) shows the requirement for cooling to temperatures below $10^{4} \mathrm{~K}$, necessary for initiate star formation (here, assuming a gas metallicity $Z=0.01 \mathrm{Z}_{\odot}$ ). (right) Same as in the left panel but for minihalos able to for stars (according to the criterion in the left panel) at redshift $z=0$ (thick curves) and $z=2$ (thin curves) and with $T_{g, \text { core }}=5000 \mathrm{~K}$.

The left panel in Figure 2 shows $n_{g, c o r e}$ and $N_{H}$ in minihalos that are able to cool to $T \sim 10^{4} \mathrm{~K}$ by $\mathrm{H}$ and He lines only. The horizontal line shows the requirement for metal cooling and star formation assuming gas metallicity $Z=0.01 \mathrm{Z}_{\odot}$. The right panel in Figure 2 shows $n_{g, c o r e}$ and $N_{H}$ for minihalos with $n_{g, c o r e}>n_{g, c o r e}^{*}$ that can cool due to hyperfine metal line transitions and may be able to form stars.

\section{Conclusions \& Discussion}

Leo T fits perfectly the model we have proposed in this contribution. Having a distance of 420 pc from the Milky Way, Leo $\mathrm{T}$ is just starting to fall into the Milky Way halo, thus it is likely to have evolved in isolation. This fulfills the first requirement of our model. Leo $\mathrm{T}$ has a bimodal star formation rate (de Jong et al. 2008) with a > 12 Gyr population and a 9 Gyr population (with low level of SF continuing to few Myrs ago), in agreement with our expectation of a late phase of accretion from the IGM. The bimodality should be most pronounced for halos with the lowest $v_{c i r}$, gradually disappearing as $v_{\text {cir }}$ increases toward the $20 \mathrm{~km} \mathrm{~s}^{-1}$ threshold that roughly defines a pre-reionization fossil (RG05). Leo $\mathrm{T}$ has a gas velocity dispersion $\sim 7 \mathrm{~km} \mathrm{~s}^{-1}$, corresponding to a dark halo with maximum circular velocity of $11 \mathrm{~km} \mathrm{~s}^{-1}$ (eq. 2.2). Leo $\mathrm{T}$ has a core radius $r_{\text {core }} \sim 100 \mathrm{pc}$, a ratio of the dynamical mass to the gas mass $M_{g} / M>100$ and maximum H I column density $N_{H}=7 \times 10^{20} \mathrm{~cm}^{-2}$. These are all in good agreement with the model (see $\S 2$ ). Sufficiently small minihalos are likely to be dark and not preenriched by the first stars. The metallicity of the gas accreted from the IGM is likely below the threshold required for initiating star formation the the halo would resemble some compact HVCs. Cosmological simulations show that the fraction of primordial minihalos that never formed stars increases with decreasing $v_{c i r}$ as $v_{\text {cir }}^{-4.6}$ and it is about $50 \%$ for halos with $v_{c i r}=10 \mathrm{~km} \mathrm{~s}^{-1}$ (Ricotti et al. 2008). Additional work is needed to determine if our model is fully consistent with the properties of the compact HVC population (Blitz et al. 1999; Braun \& Burton 1999; Robishaw et al. 2002). We predict 
maximum radii of HVCs of about $r_{h} \sim 15 \mathrm{kpc}$. This may exclude several known HVC and/or set an upper limit for their distance from the Milky Way. Larger values of $r_{h}$ are possible for halo masses $M>10^{8} \mathrm{M}_{\odot}$, a scenario already investigated by previous studied and found not fully satisfactory (Sternberg et al. 2002; Maloney \& Putman 2003; Putman et al. 2003). However, the bulk of these objects may be yet undiscovered due to their fainter H- $\alpha$ flux. The ongoing survey ALFALFA (Giovanelli et al. 2005, 2007) may be able to discover this new population of extragalactic objects.

\section{References}

Babul, A. \& Rees, M. J. 1992, MNRAS, 255, 346

Bertschinger, E. 1985, ApJS, 58, 39

Blitz, L., Spergel, D. N., Teuben, P. J., Hartmann, D., \& Burton, W. B. 1999, ApJ, 514, 818

Braun, R. \& Burton, W. B. 1999, A\&A, 341, 437

Bullock, J. S., Kolatt, T. S., Sigad, Y., Somerville, R. S., Kravtsov, A. V., Klypin, A. A., Primack, J. R., \& Dekel, A. 2001, MNRAS, 321, 559

de Jong, J. T. A., et al. 2008, ApJ, 680, 1112

Efstathiou, G. 1992, MNRAS, 256, 43P

Giovanelli, R., et al. 2005, AJ, 130, 2598

Giovanelli, R., et al. 2007, AJ, 133, 2569

Maloney, P. R. \& Putman, M. E. 2003, ApJ, 589, 270

Putman, M. E., Bland-Hawthorn, J., Veilleux, S., Gibson, B. K., Freeman, K. C., \& Maloney, P. R. 2003, ApJ, 597, 948

Ricotti, M. 2003, MNRAS, 344, 1237

Ricotti, M. \& Gnedin, N. Y. 2005, ApJ, 629, 259

Ricotti, M., Gnedin, N. Y., \& Shull, J. M. 2002, ApJ, 575, 49

Ricotti, M., Gnedin, N. Y., \& Shull, J. M. 2008, ArXiv e-prints, 802

Ricotti, M., Pontzen, A., \& Viel, M. 2007, ApJl, 663, L53

Ricotti, M. \& Wilkinson, M. I. 2004, MNRAS, 353, 867

Robishaw, T., Simon, J. D., \& Blitz, L. 2002, ApJl, 580, L129

Ryan-Weber, E. V., Begum, A., Oosterloo, T., Pal, S., Irwin, M. J., Belokurov, V., Evans, N. W., \& Zucker, D. B. 2008, MNRAS, 384, 535

Sternberg, A., McKee, C. F., \& Wolfire, M. G. 2002, ApJS, 143, 419

Wechsler, R. H., Bullock, J. S., Primack, J. R., Kravtsov, A. V., \& Dekel, A. 2002, ApJ, 568, 52 